\title{
Period-doubling bifurcation in strongly anisotropic Bianchi I quantum cosmology
}

\author{
Michael Bachmann* and Hans-Jürgen Schmidt ${ }^{\dagger}$ \\ Institut für Mathematik, Universität Potsdam, PF 601553, D-14415 Potsdam, Germany, \\ Institut für Theoretische Physik, Freie Universität Berlin, Arnimallee 14, D-14195 Berlin, Germany
}

(October 1, 2018)

\begin{abstract}
We solve the Wheeler-DeWitt equation for the minisuperspace of a cosmological model of Bianchi type I with a minimally coupled massive scalar field $\phi$ as source by generalizing the calculation of Lukash and Schmidt [1]. Contrarily to other approaches we allow strong anisotropy. Combining analytical and numerical methods, we apply an adiabatic approximation for $\phi$, and as new feature we find a period-doubling bifurcation. This bifurcation takes place near the cosmological quantum boundary, i.e., the boundary of the quasiclassical region with oscillating $\psi$-function where the WKB-approximation is good. The numerical calculations suggest that such a notion of a "cosmological quantum boundary" is well-defined, because sharply beyond that boundary, the WKB-approximation is no more applicable at all. This result confirms the adequateness of the introduction of a cosmological quantum boundary in quantum cosmology.
\end{abstract}

PACS 98.80; 98.70

\section{INTRODUCTION}

The idea to consider the whole Universe as one quantum system has already attracted many researchers. One of the approaches to give this idea a physical meaning is to consider the superspace $\mathcal{S}$ of all possible spatial 3-geometries together with the matter field degrees of freedom as supercoordinates, and the supermomentum space $\mathcal{S}^{\star}$ is formed from the corresponding second fundamental forms together with the momenta of the matter fields. The dynamics will then be defined by the Einstein field equation accompanied by the matter field equations. In the last step, this system will be quantized.

Of course, one has to deal with an infinite number of degrees of freedom already from the geometric part. The, up to now only, way to tackle such a system is to linearize in almost all degrees of freedom and to take the nonlinearities only from the remaining finitely many ones. But even this system is almost untractable. So, the idea of the minisuperspace arose: One restricts to a finite-dimensional space of spatial geometries and to matter fields with finitely many components, disregarding or even ignoring all other degrees of freedom.

From the first glance one could believe that such a big simplification would lead to a picture which has nothing to do with the real Universe's evolution. However, the results given in the last years are encouraging: The dynamics of the minisuperspace can be written as an equivalent mechanical system, and then the Schrödinger equation for this system carries the name Wheeler-DeWitt equation which can be solved for simple systems and give already a surprisingly good picture of the evolution, even for the case that the geometric part is restricted to only one degree of freedom, the "radius" of the Universe. This corresponds to the isotropic Friedmann Universe model. For this case, closed-form solutions for the Wheeler-DeWitt equation exist.

In the ordering with respect to simplicity, the next possible geometry is the Bianchi type I model, where the spatial inner geometry is flat, but the expansion is allowed to be anisotropic. For this case, we have 3 degrees of freedom

*mbach@physik.fu-berlin.de, http://www.physik.fu-berlin.de/ ${ }^{\sim}$ mbach

${ }^{\dagger}$ hjschmi@rz.uni-potsdam.de, http://www.physik.fu-berlin.de/ ${ }^{\sim}$ hjschmi 
for the geometry: the expansion rates into the 3 spatial directions. For this case, the Wheeler-DeWitt equation is already quite complicated, so one uses an approximation to solve it. One of the most powerful of these approximation schemes is the Wentzel-Kramers-Brillouin (WKB)-approximation. In Ref. [1], the first order WKB-approximation for the massive scalar field in the Bianchi type I model has been deduced, but - as far as we are aware - the higher order WKB-approximations have not yet been calculated up to now for this model. The analogous calculations for Bianchi type IX have been done by Amsterdamski [2]. In both cases, the anisotropy degrees of freedom had been assumed to be small. In the present paper we allow also large anisotropies. Ref. [3] deals with a similar model allowing large anisotropies, too; however, the authors of [3] use a simplicial minisuperspace model, so the results are not directly comparable to ours.

The text is organized as follows: Sect. II presents the geometry of the Bianchi type I cosmological model, Sect. III the corresponding Wheeler-DeWitt equation and Sect. IV its solutions both analytically and numerically. Sect. V shortly reviews Refs. [6 - 42], i.e., the earlier developments of the topic, and discusses the results.

In the appendix, we show how the different versions to solve the factor-ordering problem influence the solutions of the Wheeler-DeWitt equation.

\section{THE BIANCHI TYPE I MODEL}

The metric of a spatially flat cosmological model is deduced as follows: One requires that an abelian 3-dimensional isometry group acts transitively on 3-dimensional spatial hypersurfaces which consequently have to be flat 3-spaces. This restricts the possible metrics to

$$
d s^{2}=N^{2} d t^{2}-g_{i j} d x^{i} d x^{j}
$$

where $N=N(t)$ is the lapse function which can be put to $N=1$ by a time reparametrization, $i, j=1,2,3$, and $g_{i j}$ represents a symmetric positive definit matrix whose components depend on $t$ only. So we have 6 free components from the first consideration. However, restricting to metrics being a solution of Einstein's field equation we can simplify as follows: At any initial time, say $t=0$, we can choose the initial condition

$$
g_{i j}=\delta_{i j}=\operatorname{diag}(1,1,1) \quad \text { at } \quad t=0
$$

without loss of generality, and then by a spatial rotation not changing Eq. (2.2), the second fundamental form, i.e. $d g_{i j} / d t$, can be brought into diagonal form. By use of the Einstein equation one can show that under these circumstances, $g_{i j}$ will keep its diagonal form for all times. Thus: The Bianchi type I model containing non-diagonal terms does not represent a generalization, and we can say: Without loss of generality let

$$
g_{i j}=\operatorname{diag}\left(A^{2}(t), B^{2}(t), C^{2}(t)\right)
$$

with certain positive functions $A, B, C$.

After an obvious rearrangement of the terms we get now from Eqs. (2.1) and (2.3)

$$
d s^{2}=N^{2} d t^{2}-e^{2 \alpha}\left(e^{2 s+2 \sqrt{3} r} d x^{2}+e^{2 s-2 \sqrt{3} r} d y^{2}+e^{-4 s} d z^{2}\right)
$$

\footnotetext{
${ }^{1}$ Strictly speaking, the axially symmetric Bianchi type I model, where 2 of the 3 scale factors coincide, is even simpler, but as one will see from the calculations: restricting to axial symmetry does not really simplify the procedure.
} 
where $\alpha, r, s$ represent 3 arbitrary real functions depending on $t$. The Hubble parameter is the mean expansion, i.e. with a dot denoting $d / d t$ we get

$$
H=\frac{1}{3}\left(\frac{\dot{A}}{A}+\frac{\dot{B}}{B}+\frac{\dot{C}}{C}\right)
$$

. In the notation of Eq. (2.4), $H=d \alpha / d t$ is the Hubble parameter, and we restrict our considerations to the expanding Universe, i.e., to $H>0$. The model goes over to the spatially flat isotropic Friedmann model if the dimensionless anisotropy parameter

$$
\eta=\frac{1}{H} \sqrt{\dot{r}^{2}+\dot{s}^{2}}
$$

tends to zero.

It is essential to observe that this approach leading to the Wheeler-DeWitt equation breaks the Lorentz invariance of the system already at this level of geometry: The $3+1$ decomposition of space-time is already made from the beginning. The remaining degrees of freedom within the geometry are the following: $\tilde{t}(t)$ as arbitrary function as long as $N(t)$ remains unspecified, and $\tilde{t}(t)= \pm t$ after having fixed $N=1$. Permutations of the 3 spatial coordinates, and for the axially symmetric case also spatial rotations: If $r=0$, then a rotation in the $x y$-plane is an additional symmetry. We presented this geometric part so explicitly, because contradicting statements exist about this behavior in the literature; example: In our interpretation, $d s^{2}=d t^{2}-d x^{2}-d y^{2}-d z^{2}$ and $d s^{2}=d t^{2}-t^{2} d x^{2}-d y^{2}-d z^{2}$ represent different geometries inspite of the fact that they are locally isometric space-times.

We use a one-component real massive scalar field $\phi$ as source, and we interpret it as follows: Either it can really be such a scalar field (i.e. a spin zero field), e.g. a Higgs field which dominated the early Universe's evolution but disappeared after a symmetry breaking effect. Or, it mimics any realistic matter field in a region where spin is negligible. Let $m$ denote that mass of the scalar field. For the GUT-mediated inflationary model, one considers the following order of magnitude: $m \approx 10^{-5} m_{\mathrm{Pl}}$, where $m_{\mathrm{Pl}}$ is the Planck mass. 2

Up to now, we did not restrict the spatial coordinates $x, y$ and $z$. At the classical level (i.e. for locally solving the Einstein field equation), it makes no difference, whether they cover all the reals or whether they are cyclic ones. But already for global classical considerations it makes a difference: Supposed, $x, y$ and $z$ are cyclic, then even for the case $r=s \equiv 0$ the metric is not spatially isotropic, because the natural length of a closed spatial geodesic depends on the chosen direction. However, we get the essential difference only after quantization. If nothing different is said, we assume $x, y$ and $z$ to be cyclic with modulus 1, i.e. $x$ and $x+1$ represent the same point etc.

\section{THE WHEELER-DEWITT EQUATION}

The Wheeler-DeWitt equation is always a zero energy Schrödinger equation due to time-reparametrization invariance of the gravitational action. However, this is not really a problem, because the Schrödinger equation with non-vanishing energy can be brought to zero-energy form by a suitable shift of the potential, see e.g. Ref. [4] for a class of transformations of this type.

In the present paper, we do not consider the entropy of the system; we only want to mention the recently published result of Kleinert [5] on how the incorporation of entropy can change the model.

\footnotetext{
${ }^{2}$ In units with $c=1$, the Planck mass is about $10^{19} \mathrm{GeV}$.
} 
To formulate the equations that lead to the Wheeler-DeWitt equation [6] as easily as possible, it proves useful to apply units such that $\hbar=c=4 \pi G / 3=1$. Then the Lagrangian of the minimally coupled scalar field in Einstein's theory of gravity (see the first of Refs. [1] for details) is given by

$$
L=L_{g}+L_{m}
$$

where the gravitational part is

$$
L_{g}=\frac{1}{2 N} e^{3 \alpha}\left(\dot{r}^{2}+\dot{s}^{2}-\dot{\alpha}^{2}\right)
$$

and the matter Lagrangian is

$$
L_{m}=\frac{1}{2 N} e^{3 \alpha}\left(\dot{\phi}^{2}-m^{2} \phi^{2} N^{2}\right) .
$$

Here we supposed the lapse function $N$ to be constant in time. The minisuperspace is 4-dimensional, its coordinates are $q^{\mu}=\left(q^{0}, q^{1}, q^{2}, q^{3}\right)$ with $q^{0}=\alpha, q^{1}=\phi, q^{2}=r$, and $q^{3}=s$. The usefulness of the Misner parametrization Eq. (2.4) of the anisotropy is obvious: The kinetic part of the Lagrangian is already in diagonal form. By defining the minisuperspace metric $f_{\mu \nu}=e^{3 \alpha} \operatorname{diag}(-1,1,1,1)$ the Lagrangian (3.1) is compactly rewritten as

$$
L=\frac{1}{2 N}\left(f_{\mu \nu} \dot{q}^{\mu} \dot{q}^{\nu}-m^{2}\left(q^{1}\right)^{2} N^{2} e^{3 q^{0}}\right) .
$$

Thus the introduction of canonical momenta

$$
p_{\mu}=\frac{\partial L}{\partial \dot{q}^{\mu}}=\frac{1}{N} f_{\mu \nu} \dot{q}^{\nu}
$$

allows us to write down the classical Hamiltonian of the minisuperspace

$$
\mathcal{H}=\frac{N}{2}\left(p_{\mu} p^{\mu}+m^{2}\left(q^{1}\right)^{2} e^{3 q^{0}}\right)=0 .
$$

Time-reparametrization invariance implies $\frac{\partial \mathcal{H}}{\partial N}=0$, i.e., $\mathcal{H}=0$. The quantization in coordinate space is done as usual by going over to the appropriate operators $p_{\mu}=-i \hbar \partial / \partial q^{\mu}$ with the partial derivative $\partial$.

From now on, we will choose two different versions for the operator ordering in $p_{\mu} p^{\mu}$ :

First variant, variant A, which is the most simple one, follows the interpretation in Ref. [1] that the solutions of the forthcoming Wheeler-DeWitt equation will not essentially depend on this choice.

Second, variant B, solves the factor ordering problem by applying the supercovariance principle: It says, that the essential equations have to be covariant ones with respect to the (mini-)superspace metric. Taking variant B, we have to replace $p_{\mu}$ by $p_{\mu}=-i \hbar \nabla / \nabla q^{\mu}$ where $\nabla$ denotes the supercovariant derivative defined by $f_{\mu \nu}$.

To cover both variants simultaneously, we introduce a parameter $\epsilon$ as follows: $\epsilon=0$ for variant A and $\epsilon=1$ for variant B. Accordingly, we denote the two variants of the D'Alembert operatorf $\square_{\epsilon}$ by

$$
\square_{0}=-f^{\mu \nu} \frac{\partial^{2}}{\partial q^{\mu} \partial q^{\nu}} .
$$

and

\footnotetext{
${ }^{3}$ Here we apply the fact that the metric is always covariantly constant, so different orderings give the same result.

${ }^{4}$ The minus sign in eqs. $(3.7 / 3.8)$ is inserted to compensate for the factor $i^{2}$ in front of $p_{\mu} p^{\mu}$.
} 


$$
\square_{1}=-f^{\mu \nu} \frac{\nabla^{2}}{\nabla q^{\mu} \nabla q^{\nu}} .
$$

For the world function $\psi\left(q^{\mu}\right)$ we get the relations

$$
\square_{1} \psi=\square_{0} \psi+3 \psi_{, 0} e^{-3 \alpha}
$$

where

$$
\square_{0} \psi=e^{-3 \alpha}[\psi, 00-\psi, 11-\psi, 22-\psi, 33]
$$

The stationary Schrödinger equation with zero energy in minisuperspace $\hat{\mathcal{H}} \psi\left(q^{\mu}\right)=0$ with the wave function $\psi\left(q^{\mu}\right)$ and the Hamilton operator $\hat{\mathcal{H}}$ is called Wheeler-DeWitt equation and possesses the form

$$
\hat{\mathcal{H}} \psi\left(q^{\mu}\right)=\frac{N}{2}\left(\hbar^{2} \square_{\epsilon}+m^{2}\left(q^{1}\right)^{2} e^{3 q^{0}}\right) \psi\left(q^{\mu}\right)=0
$$

for the Bianchi type I Universe (2.4). In details, we get after dividing by $N e^{3 \alpha} / 2$

$$
0=\left(\hbar^{2}\left[\frac{\partial^{2}}{\partial \alpha^{2}}+3 \epsilon \frac{\partial}{\partial \alpha}-\frac{\partial^{2}}{\partial \phi^{2}}-\frac{\partial^{2}}{\partial r^{2}}-\frac{\partial^{2}}{\partial s^{2}}\right]+m^{2} \phi^{2} e^{6 \alpha}\right) \psi(\alpha, \phi, r, s) .
$$

In what follows we derive an approximation scheme to solve the Wheeler-DeWitt equation.

\section{SOLUTIONS OF THE WHEELER-DEWITT EQUATION}

Before we go over to solve Eq. (3.12) we want to clarify in subsection A what happens if we neglect the anisotropy from the beginning. In subsections B to D we analytically obtain solutions in a well-defined approximation, and in subsection $\mathrm{E}$ we present the results of our numerical calculation.

\section{A. Isotropic models}

For variant A, i.e., $\epsilon=0$ in Eq. (3.11) we get

$$
0=\left(\hbar^{2}\left[\frac{\partial^{2}}{\partial \alpha^{2}}-\frac{\partial^{2}}{\partial \phi^{2}}\right]+m^{2} \phi^{2} e^{6 \alpha}\right) \psi(\alpha, \phi)
$$

i.e., we simply remove all $r$ - and $s$-dependencies. For variant $\mathrm{B}$, however, this recipe does not work because the covariant derivatives mix the coordinates. After a short calculation one finds out that here also Eq. (4.1) is the correct Wheeler-DeWitt equation. The reason for this coincidence is as follows: The 2-dimensional superspace is conformally flat, and the D'Alembertian is conformally invariant, cf. the second paper in Ref. 1 for more details.

Defining $a=e^{\alpha}>0$ as new variable we have to replace $\frac{\partial}{\partial \alpha}$ by $a \frac{\partial}{\partial a}$, and we get from Eq. (4.1) now

$$
0=\left(\hbar^{2}\left[a \frac{\partial}{\partial a} a \frac{\partial}{\partial a}-\frac{\partial^{2}}{\partial \phi^{2}}\right]+m^{2} \phi^{2} a^{6}\right) \tilde{\psi}(a, \phi)
$$

a version of the Wheeler-DeWitt equation favoured in Ref. [7].

\footnotetext{
${ }^{5}$ If the dimension of the minisuperspace is $D$ instead of 4 , then the factor 3 in front of $\psi, 0$ in Eq. (3.9) will be replaced by $3(D-2) / 2$, so $\square_{0}$ and $\square_{1}$ coincide for $D=2$.
} 


\section{B. Separation ansatz}

Now we look for the solutions of Eq. (3.12). First, we make a separation ansatz

$$
\psi(\alpha, \phi, r, s)=\chi(\alpha, \phi) \cdot \rho(r) \cdot \sigma(s) .
$$

It turns out that $\rho_{, 22} / \rho=c_{2}$ and $\sigma_{, 33} / \sigma=c_{3}$ represent constants, i.e., we get

$$
\rho(r)=\rho_{1} \exp \left(\sqrt{c_{2}} r\right)+\rho_{2} \exp \left(-\sqrt{c_{2}} r\right)
$$

and

$$
\sigma(s)=\sigma_{1} \exp \left(\sqrt{c_{3}} s\right)+\sigma_{2} \exp \left(-\sqrt{c_{3}} s\right)
$$

with arbitrary constants $\rho_{i}$ and $\sigma_{i}$. For negative values $c_{2}$, the function $\rho(r)$ has a sinus-function behaviour.

One can interpret these cases as follows: If both $c_{2}$ and $c_{3}$ are negative, then we have plane waves in $r$ - and $s$-direction. This is in agreement with the fact, that a translation into $r$ - or $s$-direction can be compensated by a coordinate transformation in metric (2.4) (i.e., by multiplying $x, y$, and $z$ with suitable constants), so that all $r$ - and $s$-values should be equally probable. However, this interpretation works only in case that we allow $x, y$, and $z$ to cover all the reals. If we, however, restrict $x, y$, and $z$ to be cyclic coordinates, then this argument in favour of equal distribution of the $r$ - and $s$ - values is no more valid.

If, on the contrary, one of the constants $c_{2}$ or $c_{3}$ is non-negative, then (up to singular exceptions) the product $\rho(r) \cdot \sigma(s)$ tends to $\pm \infty$ as $r$ or $s$ does. This allows the following interpretation: The probability to have small anisotropy is exponentially small.

For the moment we keep the further interpretation open and continue the calculation. We define the constant $c_{1}$ via $c_{1}+c_{2}+c_{3}=0$ and insert Eq. (4.3) into (3.12); we get:

$$
0=\left(\hbar^{2}\left[\frac{\partial^{2}}{\partial \alpha^{2}}+3 \epsilon \frac{\partial}{\partial \alpha}-\frac{\partial^{2}}{\partial \phi^{2}}\right]+c_{1} \hbar^{2}+m^{2} \phi^{2} e^{6 \alpha}\right) \chi(\alpha, \phi) .
$$

This equation is for variant A just the Wheeler-DeWitt equation (4.1), however, now not the zero-energy equation but the equation with energy proportional to the constant $c_{1}$ representing the anisotropic degrees of freedom.

We redefine the coordinate $\alpha$ to $v=a^{3}=e^{3 \alpha}$. The coordinate $v$ is proportional to the spatial volume. Then Eq. (4.4) goes over to (after dividing by 9 )

$$
0=\left(\hbar^{2}\left[v \frac{\partial}{\partial v} v \frac{\partial}{\partial v}+\epsilon v \frac{\partial}{\partial v}-\frac{\partial^{2}}{\partial \phi^{2}}\right]+c_{1} \hbar^{2} / 9+m^{2} \phi^{2} v^{2} / 9\right) \tilde{\chi}(v, \phi) .
$$

As one can see in comparison with Eq. (4.2): Instead of the $a^{6}$ term we now have a $v^{2}$-potential.

\section{Adiabatic scalar field}

In this subsection, we assume the scalar field $\phi$ to be almost constant. Then $\gamma$ defined by $\gamma^{2}=m^{2} \phi^{2} /\left(9 \hbar^{2}\right)$ is an adiabatic constant. In Eq. (4.5) we omit the $\phi$-derivative corresponding to the adiabatic approach. To this end we consider the following equation for the exact dependence on $v$ of a new function $\hat{\chi}$

$$
\left(\hbar^{2}\left[v \frac{\partial}{\partial v} v \frac{\partial}{\partial v}+\epsilon v \frac{\partial}{\partial v}\right]+c_{1} \hbar^{2} / 9+\gamma^{2} v^{2} \hbar^{2}\right) \hat{\chi}(v, \phi)=E(\phi) \cdot \hat{\chi}(v, \phi)
$$

where $E(\phi)$ is an yet undetermined eigenvalue; here, $\phi$ plays the role of a parameter only. We define a new adiabatic constant $\Lambda$ by $\Lambda^{2}=E(\phi) \hbar^{-2}-c_{1} / 9$. Then Eq. (4.6) becomes for $\epsilon=0$ 


$$
\left(v \frac{\partial}{\partial v} v \frac{\partial}{\partial v}+\gamma^{2} v^{2}-\Lambda^{2}\right) \hat{\chi}=0
$$

Eq. (4.7) is a Bessel type ordinary differential equation. If we replace in Eq. (4.7) $\gamma v$ by $x$ and $\hat{\chi}(v, \phi)$ by $y(x, \phi)$ we get exactly Bessel's form

$$
x^{2} \frac{d^{2} y}{d x^{2}}+x \frac{d y}{d x}+\left(x^{2}-\Lambda^{2}\right) y=0,
$$

whose solutions are (the constants $C_{i}$ depend on $\Lambda$ )

$$
y=C_{1} J_{\Lambda}(x)+C_{2} J_{-\Lambda}(x) .
$$

The $J_{\Lambda}$ are called cylinder or Bessel functions and have the development

$$
J_{\Lambda}(x)=\sum_{k=0}^{\infty}(-1)^{k}\left(\frac{x}{2}\right)^{\Lambda+2 k}(k !)^{-1}(\Gamma(\Lambda+k+1))^{-1} .
$$

Now we make the ansat:

$$
\tilde{\chi}(v, \phi)=\int_{\Lambda} b_{\Lambda}(\phi) \hat{\chi}_{\Lambda}(v, \phi) d \Lambda
$$

where we assigned to $\hat{\chi}$ the subscript $\Lambda$ to emphasize $\hat{\chi}_{\Lambda}$ being an eigenfunction with eigenvalue $\Lambda$. Thus the general solution of Eq. (4.7) is written as

$$
\hat{\chi}_{\Lambda}(v, \phi)=C_{1} J_{\Lambda}(\gamma v)+C_{2} J_{-\Lambda}(\gamma v) .
$$

Inserting the ansatz (4.11) into (4.5) we get

$$
0=\int_{\Lambda}-\hbar^{2} \frac{\partial^{2}}{\partial \phi^{2}}\left[b_{\Lambda}(\phi) \hat{\chi}(v, \phi)\right]+b_{\Lambda}(\phi)\left[\hbar^{2}\left(v \frac{\partial}{\partial v} v \frac{\partial}{\partial v}+\frac{c_{1}}{9}\right)+\frac{m^{2}}{9} v^{2} \phi^{2}\right] \hat{\chi}(v, \phi) d \Lambda .
$$

In the second term of Eq. (4.13) we replace the term in the brackets [ ] by the eigenvalue $E(\phi)$ according to Eq. (4.6). $E(\phi)$ depends on $c_{2}, c_{3}$ and $\Lambda$ only, i.e., there is no $\phi$-dependence, and we write $E$ instead of $E(\phi)$

$$
0=\int_{\Lambda}-\hbar^{2} \frac{\partial^{2}}{\partial \phi^{2}}\left[b_{\Lambda}(\phi) \hat{\chi}(v, \phi)\right]+b_{\Lambda}(\phi) E \hat{\chi}(v, \phi) d \Lambda .
$$

In Eq. (4.14) we first write out the $\frac{\partial^{2}}{\partial \phi^{2}}$ applied to the product, but we assume that only $\frac{\partial^{2}}{\partial \phi^{2}} b_{\Lambda}(\phi)$ is essential, and disregarding the other terms 7 and assuming that the $\hat{\chi}$-s are all independent we obtain the simple differential equation

$$
\left[-\hbar^{2} \frac{\partial^{2}}{\partial \phi^{2}}+E\right] b_{\Lambda}(\phi)=0
$$

which possesses solutions

$$
b_{\Lambda}(\phi)=B_{1} e^{\sqrt{E} \phi / \hbar}+B_{2} e^{-\sqrt{E} \phi / \hbar} .
$$

Finally, we get the general wave function

\footnotetext{
${ }^{6}$ If only a discrete set of values $\Lambda$ appear, Eq. (4.11) will be replaced by an analogous sum.

${ }^{7}$ This is usually called adiabatic approximation with respect to $\phi$.
} 


$$
\begin{aligned}
\psi_{c_{2} c_{3}}^{(\phi)}(\alpha, \phi, r, s)= & \left(\rho_{1} \exp \left\{\sqrt{c_{2}} r\right\}+\rho_{2} \exp \left\{-\sqrt{c_{2}} r\right\}\right)\left(\sigma_{1} \exp \left\{\sqrt{c_{3}} s\right\}+\sigma_{2} \exp \left\{-\sqrt{c_{3}} s\right\}\right) \\
& \times \int_{\Lambda}\left[B_{1} \exp \left\{\sqrt{\Lambda-\left(c_{2}+c_{3}\right) / 9} \phi\right\}+B_{2} \exp \left\{-\sqrt{\Lambda-\left(c_{2}+c_{3}\right) / 9} \phi\right\}\right] \\
& \times\left[C_{1} J_{\Lambda}\left(m \phi e^{3 \alpha} / 3 \hbar\right)+C_{2} J_{-\Lambda}\left(m \phi e^{3 \alpha} / 3 \hbar\right)\right] d \Lambda
\end{aligned}
$$

with real and for the moment continuous eigenvalues $c_{2}, c_{3}$. The superscript $(\phi)$ at $\psi$ indicates that we have treated the scalar field adiabatically. This solution has to be specified by appropriate choices for the constants $\rho_{1}, \rho_{2}, \sigma_{1}, \sigma_{2}$, $B_{1}, B_{2}, C_{1}$, and $C_{2}$ from boundary conditions. Before discussing the result (4.17), however, we calculate the wave function by considering the scale factor $\alpha$ as the adiabatic variable.

\section{Adiabatic Scale Factor Approach}

Another adiabatic method to solve the Wheeler-DeWitt equation (4.4) is to treat the scale factor $\alpha$ as a slowly varying variable.

Once more, we reexpress the scale factor $\alpha$ by $v \equiv e^{3 \alpha}$, implying that we consider the Wheeler-DeWitt equation (4.5) in the following. Considering $v$ as the adiabatic variable means that we neglect derivatives with respect to $v$ in a first step. Then Eq. (4.5) becomes

$$
\left(-\hbar^{2} \frac{\partial^{2}}{\partial \phi^{2}}+\frac{\hbar^{2}}{9} c_{1}+\frac{1}{9} m^{2} \phi^{2} v^{2}\right) \hat{\chi}(v, \phi)=E(v) \hat{\chi}(v, \phi)
$$

with $E(v)$ being a still undetermined eigenvalue. By defining

$$
\omega=\frac{1}{3} m v, \quad \tilde{x}=\sqrt{\frac{\omega}{\hbar}} \phi, \quad \eta=\frac{1}{\hbar \omega}\left(E(v)-\frac{\hbar^{2}}{9} c_{1}\right)
$$

the differential equation (4.18) is simply transformed into that for the dimensionless harmonic oscillator

$$
\left[\frac{d^{2}}{d \tilde{x}^{2}}+\left(\eta-\tilde{x}^{2}\right)\right] \tilde{y}(\tilde{x})=0
$$

By substituting $x=\tilde{x}^{2}$ and then transforming $\tilde{y}(x)=e^{-x / 2} y(x)$, we eventually obtain Kummer's differential equation

$$
x \frac{d^{2} y(x)}{d x^{2}}+(\mu-x) \frac{d y(x)}{d x}-\nu y(x)=0,
$$

where in our case $\mu=1 / 2$ and $\nu=(1-\eta) / 4$. This differential equation possesses the general solution

$$
y(x)=A_{1} F_{1}(\nu, \mu ; x)+B_{1} F_{1}(\nu-\mu+1,2-\mu ; x) x^{1-\mu},
$$

expressed with the help of the confluent hypergeometric function ${ }_{1} F_{1}(a, b ; x) . A$ and $B$ are constants. Thus the partial problem of finding the solution of Eq. (4.18) is done and the result is written as

$$
\hat{\chi}_{\eta}(v, \phi)=e^{-\omega(v) \phi^{2} / 2 \hbar}\left[A_{1} F_{1}\left((1-\eta) / 4,1 / 2 ; \omega(v) \phi^{2} / \hbar\right)+B \sqrt{\frac{\omega(v)}{\hbar}} \phi_{1} F_{1}\left((3-\eta) / 4,3 / 2 ; \omega(v) \phi^{2} / \hbar\right)\right]
$$

with $\hat{\chi}_{\eta}$ denoting the eigenfunction to the real, continuous eigenvalue $\eta$.

For further processing to find an adiabatic solution for the Wheeler-DeWitt equation (4.5) we make the integral ansatz

$$
\tilde{\chi}(v, \phi)=\int_{\eta} g_{\eta}(v) \hat{\chi}_{\eta}(v, \phi) d \eta
$$


Inserting this into Eq. (4.5), we obtain (for $\epsilon=0$ )

$$
0=\int_{\eta} \hbar^{2} v \frac{\partial}{\partial v} v \frac{\partial}{\partial v}\left[g_{\eta}(v) \hat{\chi}_{\eta}(v, \phi)\right]+g_{\eta}(v)\left[-\hbar^{2} \frac{\partial^{2}}{\partial \phi^{2}}+\frac{1}{9}\left(\hbar^{2} c_{1}+m^{2} \phi^{2} v^{2}\right)\right] \hat{\chi}_{\eta}(v, \phi) d \eta .
$$

The expression enclosed in the brackets [ ] in the second term is substituted by the eigenvalue

$$
E(v)=\frac{1}{9}\left[3 \hbar m \eta v-\hbar^{2}\left(c_{2}+c_{3}\right)\right]
$$

following from Eq. (4.18) and the definitions (4.19). In contrast to the preceding approach with an adiabatic scalar field, $E(v)$ depends explicitly on the adiabatic variable which is in this case $v$. Note that $E(v)$ also depends on the eigenvalues $c_{2}, c_{3}$, and $\eta$. Now we utilize the assumption of adiabaticity with respect to $v$ by neglecting terms containing derivatives $\partial \hat{\chi}_{\eta} / \partial v$ and $\partial^{2} \hat{\chi}_{\eta} / \partial v^{2}$ which appear in the first term of Eq. (4.25). Furthermore supposing that all functions $\hat{\chi}_{\eta}(v, \phi)$ are independent with respect to $\eta$, each integrand in Eq. (4.25) vanishes. Thus we remain with solving the differential equation

$$
\left[\hbar^{2} v \frac{\partial}{\partial v} v \frac{\partial}{\partial v}+E(v)\right] g(v)=0
$$

or, more explicitly,

$$
\left[v^{2} \frac{\partial^{2}}{\partial v^{2}}+v \frac{\partial}{\partial v}+\kappa_{1} v-\frac{\kappa_{2}^{2}}{4}\right] g(v)=0
$$

with $\kappa_{1}=m \eta / 3 \hbar$ and $\kappa_{2}^{2}=4\left(c_{2}+c_{3}\right) / 9$. Applying the transformation $x=2 \sqrt{\kappa_{1} v}$ and denoting the solution as $y(x)=g(v(x))$, Eq. (4.28) takes the same Bessel form as given in Eq. (4.8), whereas the index is $\kappa_{1}$ instead of $\Lambda$ here. For this reason the general solution of Eq. (4.28) reads

$$
g_{\eta}(v)=D_{1} J_{4\left(c_{2}+c_{3}\right) / 9}(\sqrt{4 m \eta v / 3 \hbar})+D_{2} J_{-4\left(c_{2}+c_{3}\right) / 9}(\sqrt{4 m \eta v / 3 \hbar})
$$

with constants $D_{1}$ and $D_{2}$.

Thus, the complete general wavefunction obtained with an adiabatic scale factor approach (indicated by superscript $\alpha$ ) is found to be

$$
\begin{aligned}
\psi_{c_{2} c_{3}}^{(\alpha)}(\alpha, \phi, r, s)= & \left.\left(\rho_{1} \exp \left\{\sqrt{c_{2}} r\right\}+\rho_{2} \exp \left\{-\sqrt{c_{2}} r\right\}\right)\left(\sigma_{1} \exp \left\{\sqrt{c_{3}} s\right\}\right)+\sigma_{2} \exp \left\{-\sqrt{c_{3}} s\right\}\right) \\
& \times \int_{\eta}\left[D_{1} J_{4\left(c_{2}+c_{3}\right) / 9}\left(\sqrt{4 m \eta e^{3 \alpha} / 3 \hbar}\right)+D_{2} J_{-4\left(c_{2}+c_{3}\right) / 9}\left(\sqrt{4 m \eta e^{3 \alpha} / 3 \hbar}\right)\right] \exp \left\{-m \phi^{2} e^{3 \alpha} / 6 \hbar\right\} \\
& \times\left[A_{1} F_{1}\left((1-\eta) / 4,1 / 2 ; m \phi^{2} e^{3 \alpha} / 3 \hbar\right)+B \sqrt{\frac{m}{3 \hbar}} \phi e^{3 \alpha / 2}{ }_{1} F_{1}\left((3-\eta) / 4,3 / 2 ; m \phi^{2} e^{3 \alpha} / 3 \hbar\right)\right] d \eta
\end{aligned}
$$

with constants $\rho_{1}, \rho_{2}, \sigma_{1}, \sigma_{2}, A, B, D_{1}$, and $D_{2}$ to be determined via appropriate boundary conditions. The anisotropy quantum numbers $c_{2}$ and $c_{3}$ are supposed to be real at this stage. Certain boundary conditions, such as requiring the wavefunction to vanish for infinitely large values of $\alpha$ and/or $-\alpha$, lead to replacing the integral by a sum over $\eta$.

\section{E. Visualization of the world function}

Now we visualize the results of our numerical calculation of the world function (4.17). To get an impression about the shape of the probability amplitude, we concentrate on the absolute value of the wave function $|\psi|$ which is given in these six diagrams in dependence of $\alpha$, the logarithm of the cosmic scale factor, and the scalar field $\phi$. Each figure is printed at fixed values of the other parameters in Eq. 4.17). We chose $\rho_{1}=\sigma_{1}=B_{1}=C_{2}=0$ and $\rho_{2}=\sigma_{2}=B_{2}=C_{1}=1$ to ensure convergence for small and large values of $\alpha$ and $\phi$. The absolute wave function 
$|\psi(\alpha, \phi)|$ is not normalized, however, when comparing different figures (for instance Fig. 1 and Fig. 2), higher function values mean an increased probability density.

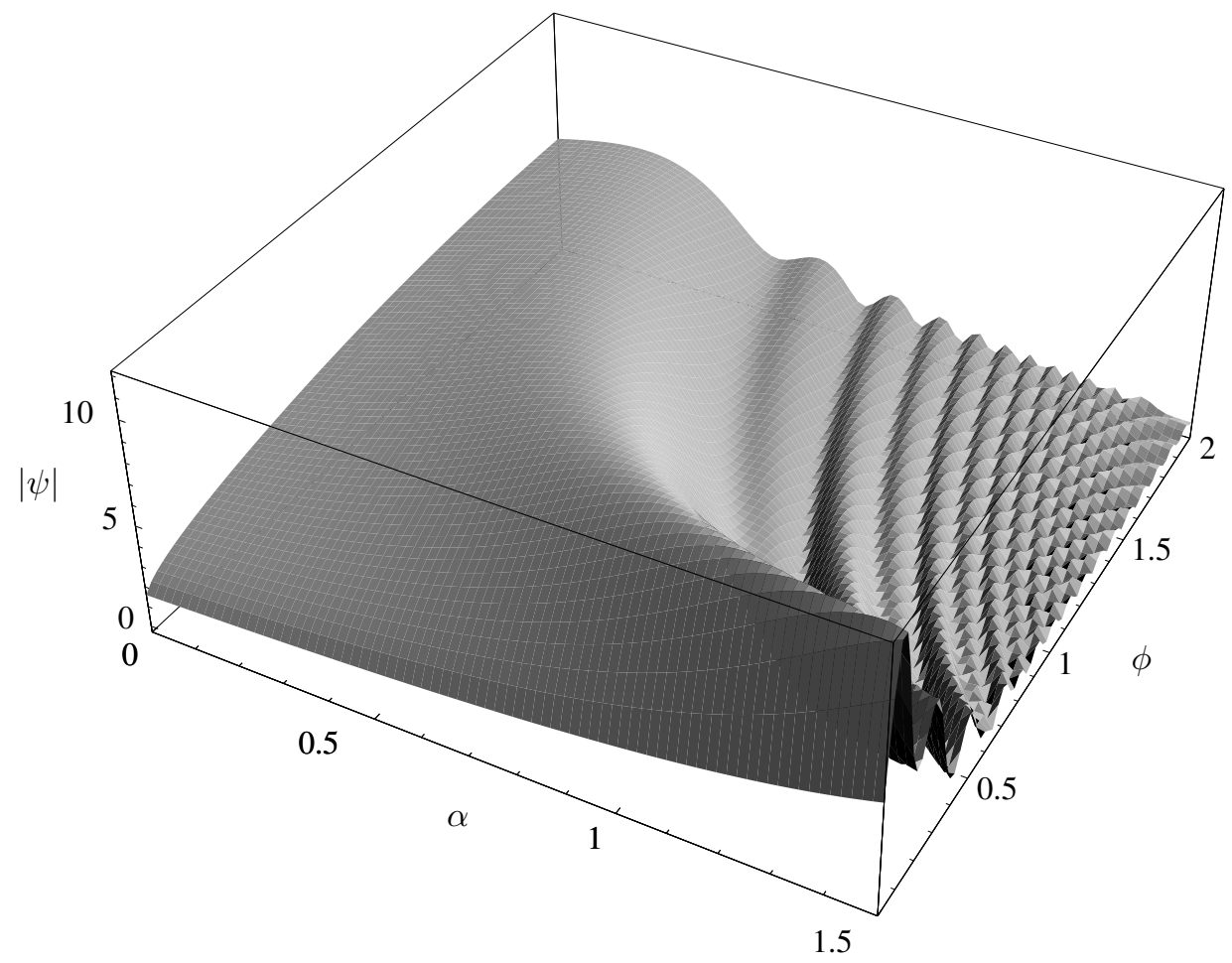

FIG. 1. The absolute value of the wave function $|\psi|$ in dependence on $\alpha$ and $\phi$ for parameters $r=s=1$ and $c_{2}=4$ and $c_{3}=7$.

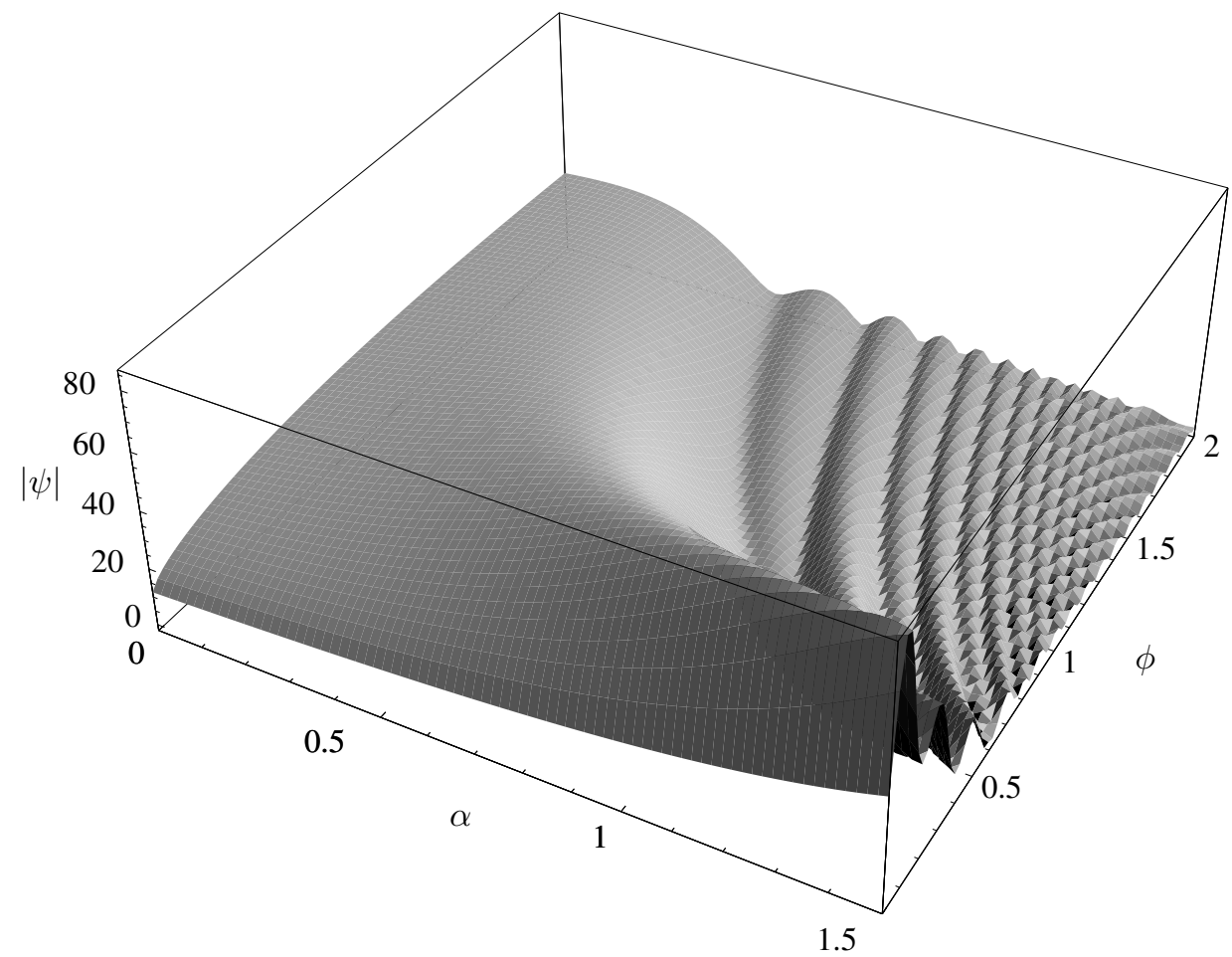

FIG. 2. The same as Fig. 1, now $c_{2}=-4$ and $c_{3}=7$. The different spacing of the $|\psi|$-values should be noted. 


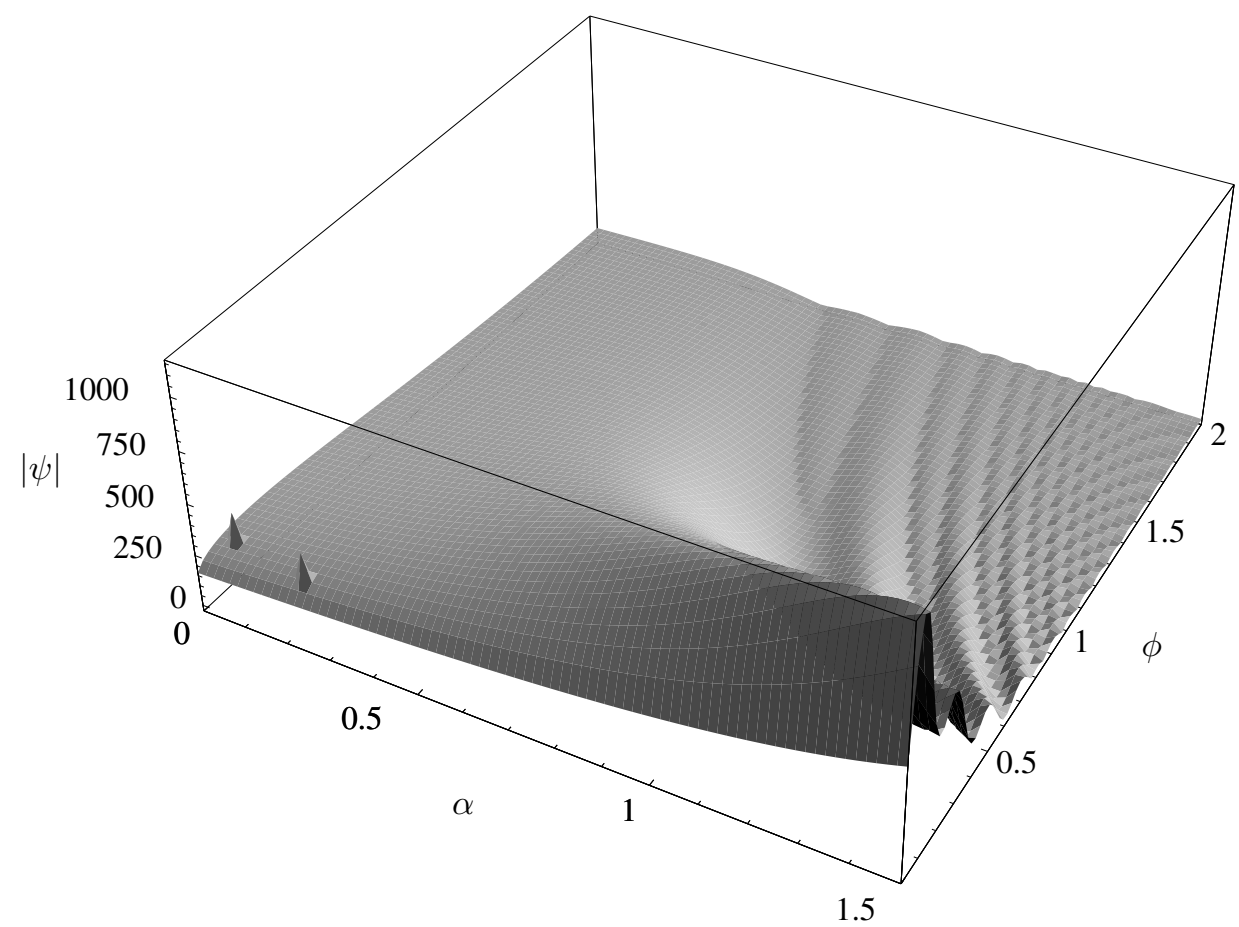

FIG. 3. The same as Fig. 1, now $c_{2}=-4$ and $c_{3}=-7$. The small discontinuities in the figure in the lower left corner may be numerical artefacts, so we do not comment them further. In comparison to Figs. 1 and 8 , the different spacing of the $|\psi|$-axis is still greater.

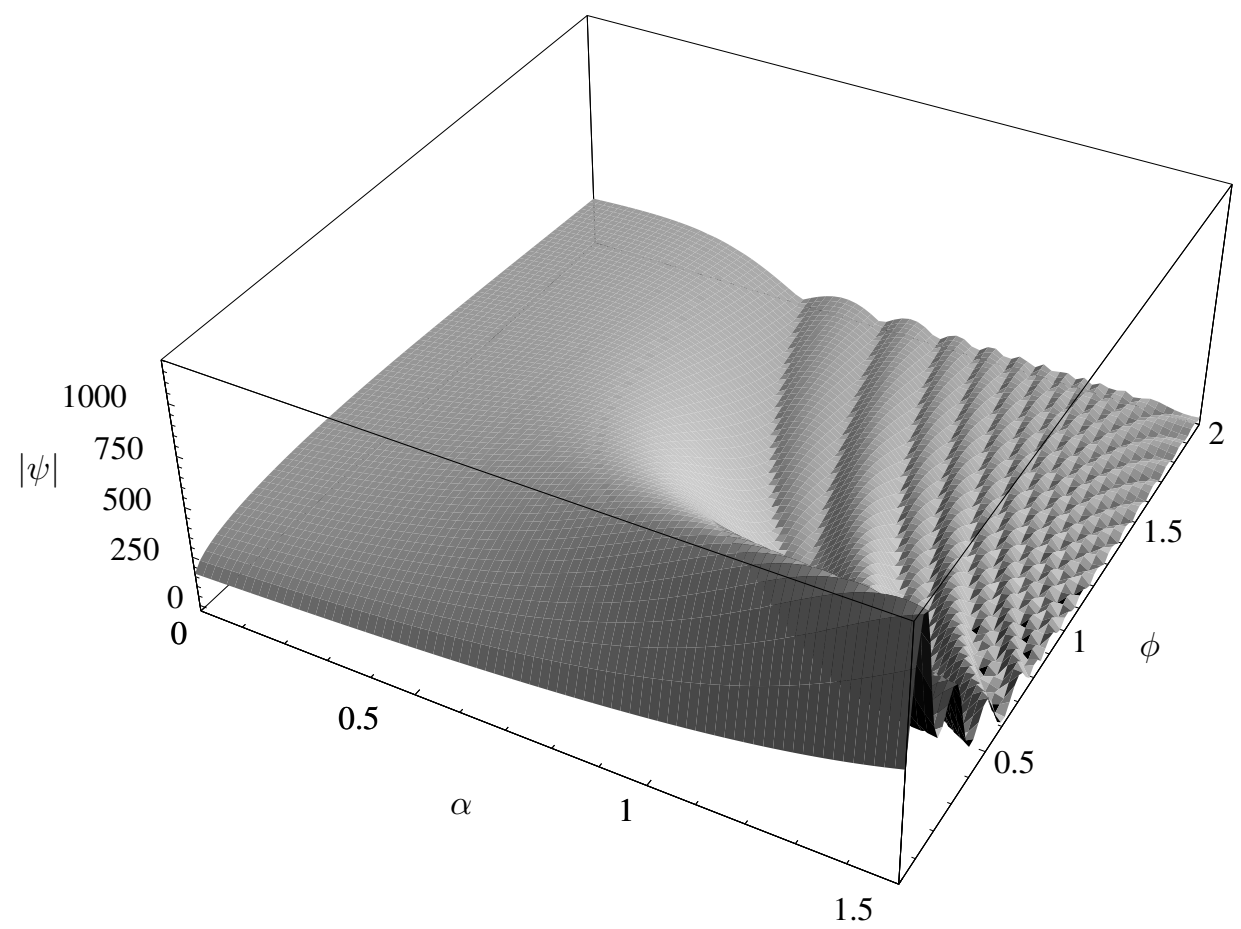

FIG. 4. The wave function in the isotropic limit, represented by $c_{2}=0$ and $c_{3}=0$. 


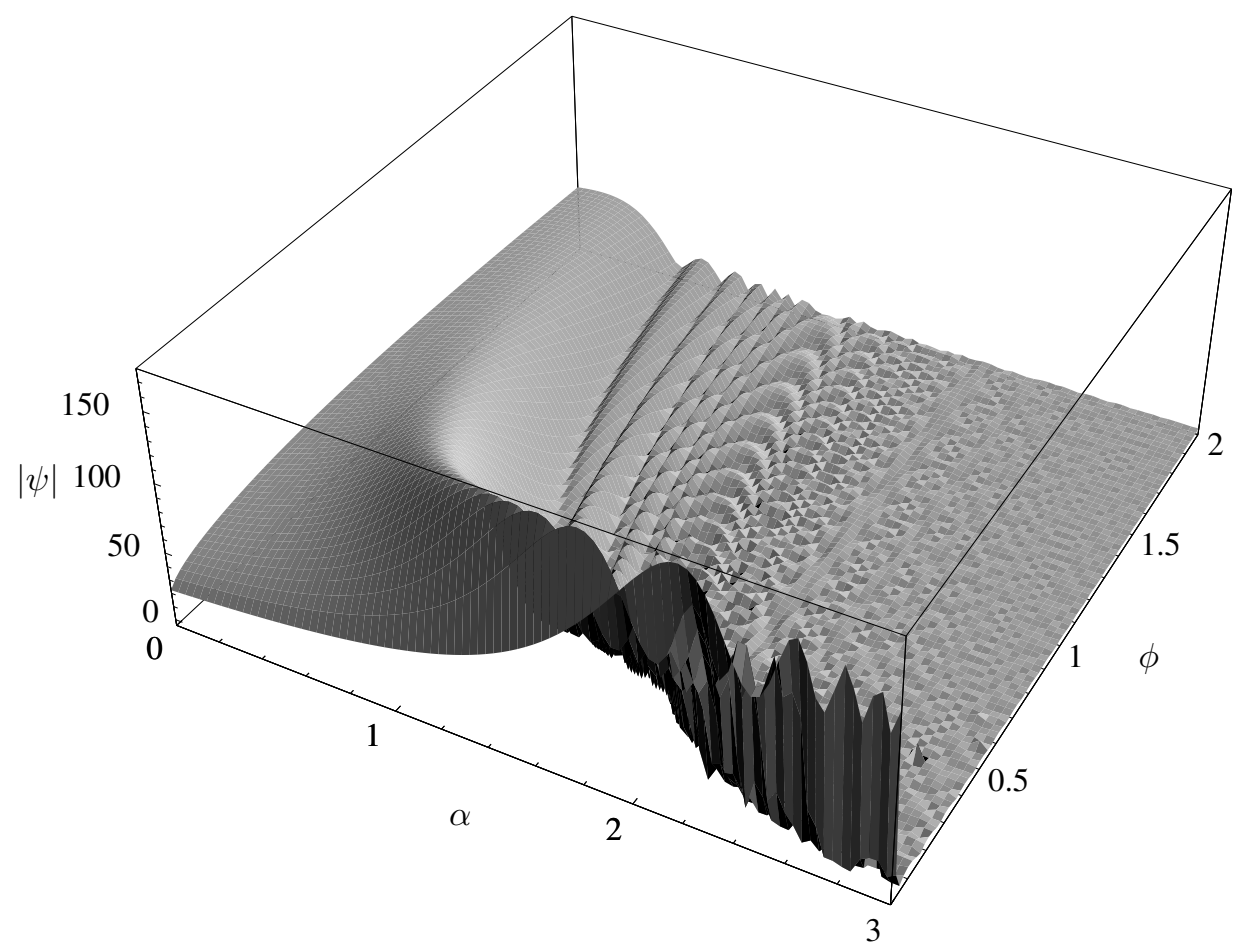

FIG. 5. The wave function with quantum numbers $c_{2}=1$ and $c_{3}=1$. This figure is also an example for the influence of the anisotropy upon the shape of the wave function.

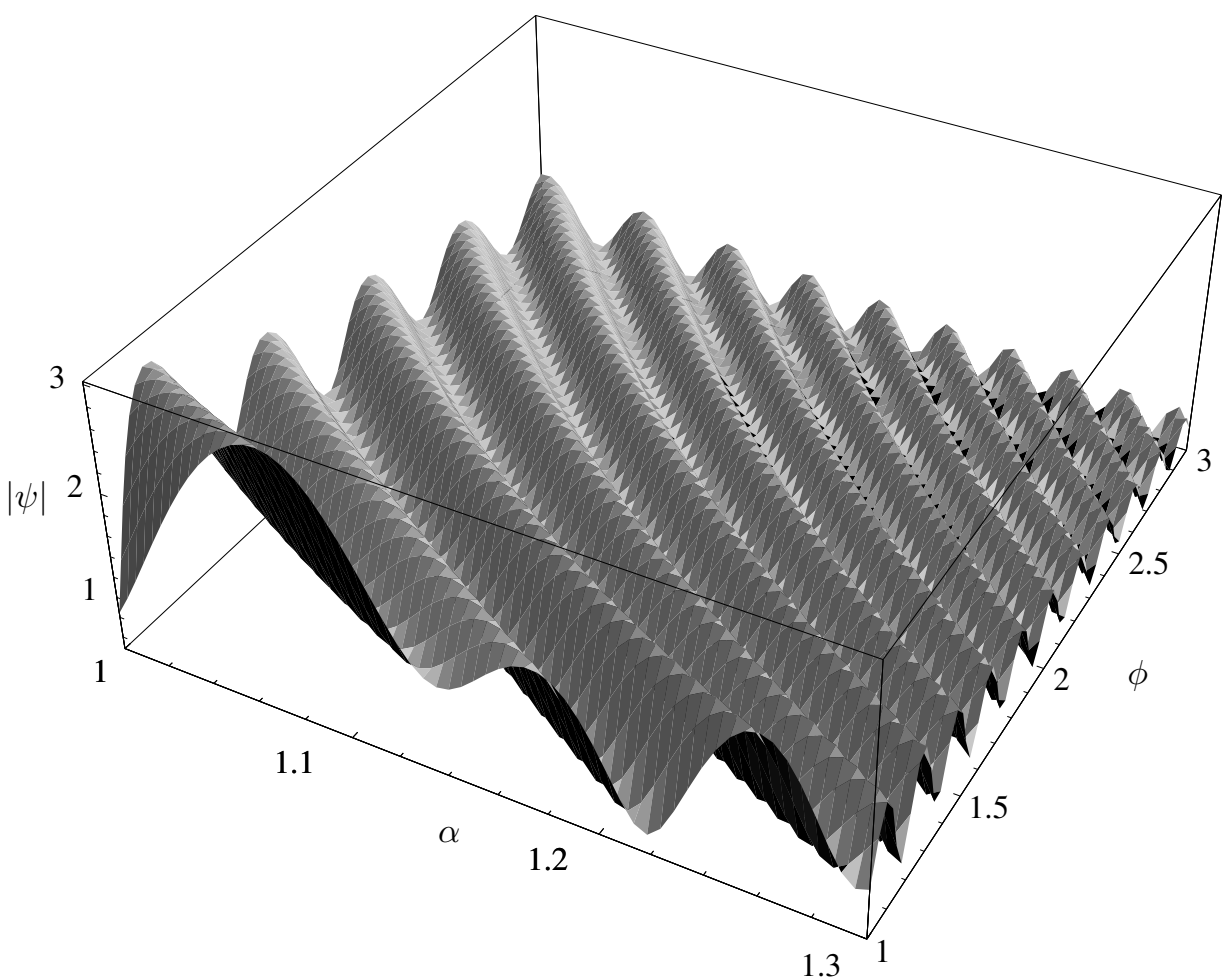

FIG. 6. Magnification of a subrange of Fig. 1. From the description it becomes clear which part of Fig. 11 represents. We show it here to make clear, how quickly the WKB-approximation becomes a good approximation.

For all these different ranges of parameters leading to the results shown in Figs. 1 15 we find essentially the same behaviour: The right part of the figure is the region where the WKB-approximation is valid, that means, the solution 
is close to a sinus-shaped wave with a slowly varying amplitude (see Fig. 6). The left hand side is the quantum region where no oscillations exist at all. The range where these two behaviours go into each other, is relatively sharply defined. This range is what one usually calls the "cosmological quantum boundary", and these pictures show up that this notion is relatively well-defined.

An interesting new feature of these figures is the following: They show a period-doubling bifurcation of the frequency if one looks from the bottom to the top of these figures with increasing values of $\phi$.

\section{DISCUSSION}

Before we discuss the results of the present paper we give a short review to other papers:

The original paper [6] by DeWitt was seminal to the whole development of quantum gravity. Its application to minisuperspace cosmological models has been developed by several researchers, e.g. the authors and editors of refs. [7] and [8]. At that time, the main discussion dealt with the closed isotropic Friedmann models, and the spatially flat Friedmann models had been included as less interesting limit cases, too.

In [9] and [10], Schön and Hajicek perform a quantization of systems with quadratic constraints and discuss the Wheeler-DeWitt equation. In ref. [11], Landsman gives details which Hilbert space might be appropriate for the isotropic minisuperspace quantization, a topic, which was not much discussed before.

In the recent preprint by Kim [12], the minimally coupled massless scalar field in an open isotropic Friedmann model has been discussed and its Wheeler-DeWitt equation be solved from the point of view that the Universe is created quantum mechanically from "nothing".

Recently, Capozziello and Lambiase [13] investigated the connection between the Hartle criterion for selecting correlated regions in the configuration space of dynamical variables and an associated Noether symmetry. This relationship serves to classify solutions of the Wheeler-DeWitt equations in semi-classical minisuperspace models. Thus, the oscillatory behaviour of a subset of solutions entails the presence of Noether symmetries which, in the consequence, select classical Universe models.

The paper [14] by Schunck and Mielke is related to the models discussed here as follows: They apply the WagonerBekenstein-Starobinsky-transformation for classifying inflationary solutions with scalar field as source, and this classification should also apply to the corresponding Wheeler-DeWitt equations.

In [15] and [16], a one-parameter set of minisuperspace metrics in arbitrary dimensions is considered, from which we have chosen only that one which gives classically the correct correspondence to Einstein's theory. In [15], the signature of the superspace metric in dependence on the signature of the underlying manifold is evaluated with the result that the normal-hyperbolic character of the Wheeler-DeWitt equation exists only for the Euclidean and the Lorentzian signature of the underlying manifold.

In [17], Horiguchi, Maeda and Sakmaoto perform an expansion of solutions of the Wheeler-DeWitt equation in powers of the Planck length. Vilenkin [18] compares several approaches to quantum cosmology, Kim and Page [19] discuss quantum Friedmann models and power-law inflation, Kim [20] compares quantum Friedmann models with conformally and minimally coupled scalar fields. In Ref. [21], Bleyer and Ivashchuk discuss multidimensional cosmological models and their corresponding Wheeler-DeWitt equations. Page [22] solves the Wheeler-DeWitt equation for scalar fields as source.

Ref. [23] represents the famous paper in which the "Hartle-Hawking boundary conditions" for the Wheeler-DeWitt equation have been derived. One takes a path integral over all such space-times $V_{4}$ whose boundary is the prescribed spatial hypersurface $V_{3}$. 
In Ref. [24], Kiefer constructs wavepackets in minisuperspace for a Friedmann Universe. An adiabatic approach is used in the case of a massive scalar field, thereby assuming the scale parameter $a$ to be slightly changing only. In Zeh [24], these solutions are discussed under the point of view of the definition of the direction of time. Our Eq. (4.1) coincides with the form discussed in Ref. [24] (Eq. (6.5) of Zeh, and Eq. (2.2) of Kiefer). The approximation of our subsection IV D was used by Kiefer [24] for solving the Wheeler-DeWitt equation of a Friedmann Universe. In contrast to Kiefer's procedure, where the harmonic oscillator has discrete eigenvalues and thus the wave function decreases for large values of the scale factor $\alpha$, we allow all real eigenvalues.

Conradi [25] solves the Wheeler-DeWitt equation for Bianchi type IX and a massive scalar field.

Grishchuk and Sidorov [26] discuss the initial conditions for the Wheeler-DeWitt equation, especially for the massive scalar field in a closed Friedmann model.

In Ref. [27], Amendola, Khalatnikov, Litterio, and Occhionero consider quantum cosmology with a complex field.

Guendelmann and Kaganovich [28] discuss cosmic time in quantum cosmology. The factor-ordering problem is solved such that the kinetic term gets the form $\square+\xi R$ where $\xi \leq \xi_{\text {conf }}$.

A comparison of the minisuperspace of minimally and conformally coupled scalar fields was done by Page [29]. He solves the factor-ordering problem of the Wheeler-DeWitt equation by requiring that the kinetic term is proportional to the Laplacian in the minisuperspace metric, i.e., our variant B with $\epsilon=1$. The classical equation is similar to the geodesic equation in superspace.

Refs. [30] deal with quantum cosmology from the path integral point of view: The Wheeler-DeWitt equation can be derived in a first approximation from the corresponding path integral. Halliwell [30] solves the factor-ordering problem by requiring invariance with respect to field redefinition of both 3-metric and lapse function. Jafariztadeh, Darabi and Rastegar [30] apply the method of Duru and Kleinert to evaluate the path integral for quantum cosmology, cf. Kleinert [30].

DeWitt [30] assumes the path integral to be the more fundamental approach, but the Wheeler-DeWitt equation in the minisuperspace to remain a good approximation to it.

By considering the Wheeler-DeWitt equation

$$
\left(a^{-p} \frac{\partial}{\partial a} a^{p} \frac{\partial}{\partial a}+a^{4} \frac{\Lambda}{3}\right) \psi(a)=0
$$

Gibbons and Grishchuk [31] obtain the result that inflation is typical in the set of spatially flat Friedmann models in Einstein's theory with a $\Lambda$-term. The parameter $p$ is due to the factor-ordering ambiguity, they take $p=1$ as a preferred value. An analogous result is given by Hawking and Page [32]. Melnikov and Pevcov [33] discuss the factorordering problem for the Wheeler-DeWitt equation in closed and open Friedmann models and give some solutions to it.

Reuter and Schmidt [34] and Schmidt [35] derive the Wheeler-DeWitt equation of fourth-order gravity for a spatially flat Friedmann model and compare with the corresponding conformally equivalent (due to the Bicknell theorem) second order models. The solution of Reuter/Schmidt for flat Friedmann models is generalized in Ref. [36] by Pimentel and Obregon to closed and open models. Fabris and Reuter [36] continue to generalize the results of [34] to show that the Bicknell theorem applies also at the level of the Wheeler-DeWitt equation.

Rainer [37] gives an overview on higher dimensions and discusses 3 types of conformal transformations of different levels for the Wheeler-DeWitt equation. Quite recently, the solutions of the Wheeler-DeWitt equation in comparison with the appearance of singularities is treated by Mongan [38]. Zhang and Shen [39] consider quantum cosmology with a complex scalar field at finite temperature. 
A critical discussion of the Wheeler-DeWitt equation and an alternative quantization scheme is presented in Ref. [40] by Peres.

From recent constraint calculations of Hwang and Noh [41] to an inflation model based on a non-minimally coupled massive scalar field and comparisons with observational data (COBE-DMR), one can state that minimal coupling is a good approximation for inflationary models. Following Futamase and Maeda [42], the coupling constant is either quite small, $\xi<1 / 1000$, or negative. According to the coupling factor $\left(1-\xi \phi^{2}\right)$ in front of $R$, critical behaviour appears for $\xi=\phi^{-2}$, i.e. for positive values of $\xi$ only.

Let us now summarize our results: We solved the Wheeler-DeWitt equation for the minisuperspace of a cosmological model of Bianchi type I with a minimally coupled massive scalar field $\phi$ as source by generalizing the calculation of Lukash and Schmidt [1]. Contrarily to other approaches we allowed strong anisotropy.

Combining analytical and numerical methods, we applied an adiabatic approximation for $\phi$, and as new feature we found a period-doubling bifurcation of the typical solutions. This bifurcation takes place near the cosmological quantum boundary, i.e., the boundary of the quasiclassical region with oscillating $\psi$-function where the WKBapproximation is good. The numerical calculations suggest that such a notion of a "cosmological quantum boundary" is well-defined, because sharply beyond that boundary, the WKB-approximation is no more applicable at all.

This result confirms the adequateness of the introduction of a cosmological quantum boundary in quantum cosmology. We applied the supercovariance principle, i.e., the underlying theory should also be covariant with respect to transformations representing a mixture between space-time and matter degrees of freedom. With our figures, we tried to vizualize the birth of the Universe.

\section{ACKNOWLEDGEMENT}

One of us (M.B.) is supported by the Studienstiftung des deutschen Volkes. H.-J. S. gratefully acknowledges financial support from DFG (KL 256/31-2). We thank the colleagues of the Free University Berlin, where this work has been done, especially Prof. H. Kleinert and Dr. A. Pelster, for kind hospitality, and Dr. A. Kirillov for valuable comments.

\section{APPENDIX: VARIANT A VS. VARIANT B}

Until Eq. (4.6) we parallely dealt with variant A $(\epsilon=0)$ and $\mathrm{B}(\epsilon=1)$, but beginning from Eq. (4.7) we simplified by restricting to variant $\mathrm{A}$. Now we want to complete the calculation by showing what changes using variant $\mathrm{B}$.

The purpose of this appendix is to show in detailed calculations what has been verbally mentioned in the literature several times, namely the fact that differences in solving the factor-ordering problem do not essentially change the results. For this consideration we now set $\epsilon=1$ in Eq. (4.6) and use the definitions below that equation:

$$
\left(\left[v \frac{\partial}{\partial v} v \frac{\partial}{\partial v}+v \frac{\partial}{\partial v}\right]+\gamma^{2} v^{2}-\Lambda^{2}\right) \hat{\chi}(v, \phi)=0 .
$$

The transformation from Eq. (4.7) to Eq. (4.8) now leads to

$$
x^{2} \frac{d^{2} y}{d x^{2}}+2 x \frac{d y}{d x}+\left(x^{2}-\Lambda^{2}\right) y=0
$$

whose solutions are also Bessel functions, modified by a factor $x^{-1 / 2}$ :

$$
y(x)=x^{-1 / 2}\left[\tilde{C}_{1} J_{\sqrt{\Lambda^{2}+1 / 4}}(x)+\tilde{C}_{2} J_{-\sqrt{\Lambda^{2}+1 / 4}}(x)\right]
$$


where $\tilde{C}_{1}, \tilde{C}_{2}$ are constants.

This means that the wavefunction (4.17) using variant $\mathrm{A}$ is changed by multiplying by a factor $\sqrt{3 \hbar e^{-3 \alpha} / m \phi}$. Moreover, the index $\Lambda$ of the Bessel functions must be replaced by $\sqrt{\Lambda^{2}+1 / 4}$.

\section{REFERENCES}

[1] V. Lukash, H.-J. Schmidt, Astron. Nachr. 309, 25 (1988); H.-J. Schmidt, J. Math. Phys. 37, 1244 (1996); gr-qc/9510062.

[2] P. Amsterdamski, Phys. Rev. D 31, 3073 (1985).

[3] C. Da Silva and R. Williams, Class. Quant. Grav. 16, 2681 (1999).

[4] A. Pelster, Dissertation Univ. Stuttgart, Germany, (1996).

[5] H. Kleinert, Phys. Lett. B 460, 36 (1999).

[6] B. DeWitt, Phys. Rev. 160, 1113 (1967).

[7] L. P. Grishchuk and Yu. V. Sidorov, p. 700 in: Proc. 4th Sem. Quantum Gravity, eds.: M. Markov, V. Berezin, and V. Frolov (World Scientific, Singapore, 1988).

[8] D. N. Page, p. 82 in: Proc. 5th Sem. Quantum Gravity, eds.: M. Markov, V. Berezin, and V. Frolov (World Scientific, Singapore, 1991).

[9] M. Schön and P. Hajicek, Class. Quant. Grav. 7, 861 (1990).

[10] P. Hajicek, Class. Quant. Grav. 7, 871 (1990).

[11] N. Landsman, p. 256 in: Current Topics in Mathematical Cosmology, eds.: M. Rainer, H.-J. Schmidt (World Scientific, Singapore 1998).

[12] S. P. Kim, Preprint gr-qc/9909002 (1999).

[13] S. Capozziello, G. Lambiase, Selection rules in minisuperspace quantum cosmology, Preprint (1999), Gen. Relat. Grav. 32 (2000) in press.

[14] F. Schunck, E. Mielke, Phys. Rev. D 50, 4794 (1994).

[15] H.-J. Schmidt, p. 405 in: Differential Geometry and Applications, eds.: J. Janyska, D. Krupka (World Scientific, Singapore, 1990).

[16] S. Odintsov, I. Ševčenko, Isv. Vuzov Fizika (Univ. Tomsk), 7, 74 (1991); D. Giulini, What is the geometry of superspace?, Preprint gr-qc/9311017 (1993).

[17] T. Horiguchi, K. Maeda and M. Sakmaoto, Analysis of the Wheeler de Witt equation beyond Planck scale, Preprint hep-th/9409152 (1994).

[18] A. Vilenkin, Phys. Rev. D 50, 2581 (1994).

[19] S. Kim and D. Page, Phys. Rev. D 45, R3296 (1992).

[20] S. Kim, Phys. Rev. D 46, 3403 (1992).

[21] U. Bleyer and V. Ivashchuk, Phys. Lett. B 332, 292 (1994).

[22] D. Page, in: Quantum concepts in space and time, p.274 eds.: C. Isham, R. Penrose (Oxford Univ. Press 1985).

[23] J. Hartle and S. Hawking, Phys. Rev. D 28, 2960 (1983).

[24] C. Kiefer, Phys. Rev. D 38, 1761 (1988); H. Zeh, The physical basis of the direction of time (Springer-Verlag Berlin 1992).

[25] H. Conradi, Phys. Rev. D 46, 612 (1992).

[26] L. Grishchuk and Ju. Sidorov, J. eksp. i teor. Fiz. 94, 29 (1988).

[27] L. Amendola, I. Khalatnikov, M. Litterio and F. Occhionero, Phys. Rev. D 49, 1881 (1994).

[28] E. Guendelmann, A. Kaganovich, Int. J. Mod. Phys. D 2, 221 (1993).

[29] D. Page, J. Math. Phys. 32, 3427 (1991). 
[30] J. Halliwell, Phys. Rev. D 38, 2468 (1988); H. Kleinert, Path integrals in Quantum Mechanics, Statistics, and Polymer Physics, 2nd ed. (World Scientific, Singapore, 1995); M. Jafariztadeh, F. Darabi, A. Rastegar, On Duru-Kleinert path integral in quantum cosmology, Preprint gr-qc/9811080 (1998); B. DeWitt, p. 6 in: Proc. 8th M. Grossmann Meeting Jerusalem, ed.: T. Piran (World Scientific Singapore 1999).

[31] G. Gibbons and L. Grishchuk, Nucl. Phys. B 313, 736 (1989).

[32] S. Hawking and D. Page, Nucl. Phys. B 264, 185 (1986).

[33] V. Melnikov and G. Pevcov, Izv. Vuzov Fizika (Tomsk) 4, 45 (1985).

[34] S. Reuter and H.-J. Schmidt, p. 243 in: Proc. 5th Intern Conf. Differential Geometry and Appl., eds.: O. Kowalski, D. Krupka (Silesian University Opava 1993).

[35] H.-J. Schmidt, Phys. Rev. D49, 6354 (1994), Erratum D54 (1996) 7906.

[36] L. Pimentel, O. Obregon, Class. Quant. Grav. 11 (1994) 2219; J. Fabris, S. Reuter, Gen. Relat. Grav. 32 (2000) in press.

[37] M. Rainer, Grav. and Cosmol. 1, 121 (1995).

[38] T. Mongan, Gen. Relat. Grav. 31, 1429 (1999).

[39] T. Zhang, Y. Shen, Int. J. Theor. Phys. 38, 1969 (1999).

[40] A. Peres, Critique of the Wheeler-DeWitt equation, p. 367 in: On Einstein's path, Essays in honour of E. Schücking, ed. A. Harvey (Springer-Verlag Berlin 1999).

[41] J. Hwang, H. Noh, Preprint astro-ph/9908340 (1999), Phys. Rev. D in print.

[42] T. Futamase and K. Maeda, Phys. Rev. D 39, 399 (1989). 